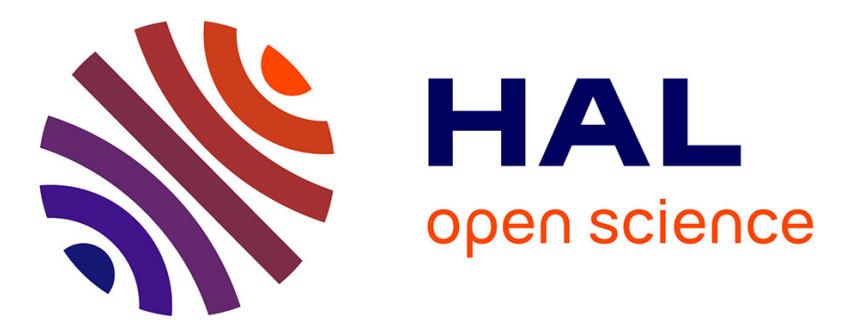

\title{
Le laboratoire cévenol de l'après-mine. Une coextensivité des causes et des responsabilités minières, environnementales et sanitaires
}

Béatrice Mésini

\section{- To cite this version:}

Béatrice Mésini. Le laboratoire cévenol de l'après-mine. Une coextensivité des causes et des responsabilités minières, environnementales et sanitaires. Géocarrefour - Revue de géographie de Lyon, 2018, Visibilité et invisibilité de la pollution des sols dans les territoires (post)industriels: de nouvelles perspectives sur la résilience et la justice environnementale ?, 92 (92/2), https://journals.openedition.org/geocarrefour/11887. 10.4000/geocarrefour.11887 . hal-01991941

\section{HAL Id: hal-01991941 \\ https://hal-amu.archives-ouvertes.fr/hal-01991941}

Submitted on 25 Apr 2019

HAL is a multi-disciplinary open access archive for the deposit and dissemination of scientific research documents, whether they are published or not. The documents may come from teaching and research institutions in France or abroad, or from public or private research centers.
L'archive ouverte pluridisciplinaire HAL, est destinée au dépôt et à la diffusion de documents scientifiques de niveau recherche, publiés ou non, émanant des établissements d'enseignement et de recherche français ou étrangers, des laboratoires publics ou privés.

\section{(이) $\$$}

Distributed under a Creative Commons Attribution - NonCommercial - NoDerivatives 4.0 


\section{Géocarrefour}

$92 / 2 \mid 2018$

Visibilité et invisibilité de la pollution des sols dans les territoires (post)industriels : de nouvelles perspectives sur la résilience et la justice environnementale?

\section{Le laboratoire cévenol de l'après-mine.Une coextensivité des causes et des responsabilités minières, environnementales et sanitaires}

The Cevennes laboratory of the post-mine. A coextensivity of mining, environmental and sanitary causes and responsibilities

\section{Béatrice Mésini}

\section{(2) OpenEdition}

Journals

Édition électronique

URL : http://journals.openedition.org/geocarrefour/11887

DOI : $10.4000 /$ geocarrefour. 11887

ISSN : 1960-601X

Éditeur

Association des amis de la Revue de géographie de Lyon

Ce document vous est offert par Aix-Marseille Université (AMU)

\section{(Aix Marseille \\ universite}

Référence électronique

Béatrice Mésini, «Le laboratoire cévenol de l'après-mine.Une coextensivité des causes et des responsabilités minières, environnementales et sanitaires », Géocarrefour [En ligne], 92/2 | 2018, mis en ligne le 25 décembre 2018, consulté le 25 avril 2019. URL : http://journals.openedition.org/ geocarrefour/11887; DOI : 10.4000/geocarrefour.11887

Ce document a été généré automatiquement le 25 avril 2019

(c) Géocarrefour 


\title{
Le laboratoire cévenol de l'après- mine.Une coextensivité des causes et des responsabilités minières, environnementales et sanitaires
}

\author{
The Cevennes laboratory of the post-mine. A coextensivity of mining, \\ environmental and sanitary causes and responsibilities
}

Béatrice Mésini

1 Le périmètre de notre étude se focalise sur deux villages ruraux de Saint-Félix-dePallières et de Thoiras localisés à l'Ouest d'Anduze, dont les populations partagent les nuisances et désordres miniers, en raison de la connexité de cinq anciennes concessions ${ }^{1}$ de la société belge des mines et fonderies de zinc de la Vieille-Montagne. Après l'apogée de l'industrie des métaux non ferreux, le déclin brutal du secteur a progressivement effacé les signes et traces de l'activité minière dans la région champêtre et touristique d'Anduze. Les néo-ruraux ont repeuplé ces campagnes désertifiées dans les années 1970 et 1980, en développant une intense pluriactivité sociale, agricole, culturelle, artisanale par l'entremise d'associations variées, avant que la parution d'un rapport du BRGM en 2008 ne dévoile la persistance et rémanence de la pollution des milieux naturels habités et cultivés. La matérialité des risques miniers a pris corps dans le hameau de Pallières, après l'effondrement brutal d'une dalle d'obturation d'un puits en octobre 2012.

Le concept de résilience s'entend comme l'aptitude des systèmes à faire face à l'aléa et au risque, en apprenant du passé, en évaluant les menaces, en supervisant les développements à court terme et en anticipant les menaces à long terme (Reghezza-Zitt et al., 2012, p. 22). En favorisant la diversité socio-culturelle et la pluriactivité agrienvironnementale dans des campagnes en déprise, nous faisons l'hypothèse que ce terreau associatif favorise des formes de résistance et de résilience chez les populations affectées. Plusieurs questions traversent l'analyse : est-ce qu'à travers leurs conduites et pratiques de vie, les habitants de ces sites pollués supportent de manière 
disproportionnée, ou font-ils supporter aux autres les risques toxiques des pollutions héritées ? Les inégalités d'exposition aux contaminants redoublent-elles des inégalités de traitement dans la prise en compte des dommages miniers (Christen et Hamman, 2008) ? Dans quelle mesure les associations organisées sur la préservation de l'environnement et de l'humain, favorisent-elles la "production d'une communauté de gestion des risques " (Le Blanc et al., 2009)?

Pour y répondre, les débats et entretiens avec les résidents du secteur minier PallièresGravouillère et ceux venus d'autres sites pollués de la région ont été enregistrés lors d'une rencontre co-organisée avec l'association HALEM en août $2016^{2}$, puis retranscrits dans le cadre d'un projet ANR ${ }^{3}$ portant sur l'effort et les inégalités environnementales. Les témoignages recueillis attestent de l'ampleur de la controverse, en révélant les sentiments ambivalents d'incrédulité, de contestation, de révolte et de combat qui animent les habitants face au dévoilement des risques. Puis le dépouillement des très nombreux rapports et dossiers dédiés ont permis de se familiariser avec cette histoire des mines en Cévennes, en décryptant le langage juridico-technique des acteurs industriels, des institutionnels et des politiques, en appréhendant la conflictualité de l'après-mine dans les médias et les sites associatifs dans un contexte de forte publicisation des risques et dommages locaux.

Dans un premier temps, nous retracerons la socio-histoire de l'implantation des mines sur ce piémont cévenol, moteur de développement depuis la fin du XIX ${ }^{\mathrm{e}}$ siècle jusqu'au lendemain de la seconde Guerre Mondiale, suivi d'un déclin amorcé depuis les années 1970 dans l'indifférence générale, car les aides publiques à la revitalisation ont été « parcimonieuses et largement inefficaces" (Vaulont et Vo Van Qui, 2011, p. 82). Dans un second temps, nous montrerons comment l'apport démographique et le renouveau associatif ont su enrayer le déclin de ces communes rurales sinistrées, en raison d'une accessibilité économique et foncière aux biens immobiliers. Pour finir, nous examinerons l'imprévisibilité de la gouvernance "glocale", dans un cadre politique et juridique complexe de missions cloisonnées et de partage indécis des responsabilités entre l'État, l'industriel, les élus, les institutions. En contrepoint, nous creuserons la question des inégalités environnementales, saillante lorsqu'elle elle met aux prises les dangers et les diverses façons d'y faire face, en révélant l'exposition, la vulnérabilité et la résilience des populations affectées (Larrère, 2017).

\section{Socio-histoire d'une industrie minière en Cévennes gardoises}

5 Le Gard est une région qui bénéficie d'un environnement géologique à forte teneur en arsenic, plomb, antimoine et cadmium. Le début de l'exploitation minière date de l'époque romaine, sachant que le gisement de la Croix-de-Pallières et PallièresGravouillère a donné lieu à trois grandes périodes d'exploitation : 1844-1888, 1911-1931 et 1948-1971.

\section{Une expansion spatiale et économique des concessions minières}

Instituée pour exploiter la pyrite de fer en 1809, la concession de "Pallières » a été développée par Jules Mirial, qui construisit une usine de vitriol sur la commune de 
Thoiras. Puis la concession « Pallières et Gravouillère » s'est élargie au sud en 1822 pour produire de l'acide sulfurique. En 1844, lorsque l'exploitation minière a pris de l'ampleur suite à la découverte d'amas de blende (zinc) et de galène (plomb) dans la région, Jules Mirial organise la Société des Mines et Usines de Pallières qui devient propriétaire de la concession de la Croix-de-Pallières en 1848, pour le plomb, le zinc, l'argent et métaux connexes, sur une superficie de 1048 hectares.

7 La Société absorbe ensuite la concession de «Pallières et Gravouillère », puis étend son emprise territoriale sur d'autres communes, en achetant ou amodiant les concessions de «La Coste» en 1847 (sur la commune Durfort), de «Valensole» en 1858 (sur les communes de Tornac et Saint-Martin-de-Sossenac), puis de "Valleraube " (sur la commune Saint-Félix de Pallières) en 1965. À partir de 1883, l'exploitation des différentes mines cesse en raison de l'importation du soufre de Sicile, qui engendre une concurrence importante dans l'extraction de la pyrite de fer (Grasset, 2017).

8 Entreprise belge spécialisée dans l'extraction et le traitement du zinc et du plomb argentifère la Société des mines et fonderies de zinc de la Vieille Montagne réinvestit le site en 1837, en amodiant trois concessions (en 1913 et 1922), puis en construisant une usine de traitement du minerai en 1926 (broyage et concentration). Après sa fermeture en 1971, le site a été racheté par la société Umicore, multinationale belge leader de la récupération des métaux précieux, qui entamé une réhabilitation du site en 1994-95 avec l'objectif de revendre les parcelles. Le traitement des métaux a produit près de deux millions de tonnes de déchets toxiques, des fines de mines dont la moitié constitue la principale digue à stériles, le reste ayant été déposé pour remblayer, mais aussi enfoui selon les témoignages des ancien mineurs (Renon et Jouve, 2016).

Suite à la cessation de l'activité minière, la renonciation a été acceptée par arrêté ministériel concernant les concessions minières de «La Croix-de-Pallières » par arrêté préfectoral du 19 mars 2004, « Pallières et Gravouillère » (18 mai 2004) et « Valleraube » (24 avril 2005). Si la mine témoin d'Alès et la maison du mineur de la Grand'Combe conservent la mémoire des mines en Cévennes, tel n'est pas le cas de la ville touristique d'Anduze qui promeut la richesse de son patrimoine, la beauté de ses paysages, la diversité de ses chemins de randonnée, la richesse de ses terroirs. Autre signe révélateur, la charte du parc naturel des Cévennes de 2007 ne consacre que quatre mentions au passé minier, les élus se montrant soucieux de lutter contre la déprise rurale, en accompagnant la reconversion et renaturalisation des sites.

Le rôle des déchets miniers et la patrimonialisation des vestiges ont été étudiés dans « l'articulation du passé et du présent» (Bretesché et Ponnet 2013, Fontaine 2016), mais quel futur concevoir pour les sites pollués dont les restes ont disparu, lorsque les terrains d'exploitation ont été rachetés puis «remis en valeur» par leurs nouveaux propriétaires?

\section{Effacement des restes d'exploitation, la tentation d'amnésie}

11 Les aménagements nécessaires à l'exploitation ont été implantés sur les deux communes de Saint-Félix-de-Pallières où se trouvaient le puits $n^{\circ} 1$, le transformateur, la salle du personnel et magasin, le hangar des fours, et de Thoiras qui accueillait la laverie, les puits $\mathrm{n}^{\circ} 2, \mathrm{n}^{\circ} 3, \mathrm{n}^{\circ} 3$ bis, la cantine, le laboratoire, le concassage, les douches, la lampisterie, les ateliers, les bureaux, la salle des pompes. Selon le mémoire de la société Umicore de juillet 1998, il ne subsiste rien de l'ancien carreau de la mine et équipements, le dépôt de 
stérile a été réhabilité par le reprofilage du plateau par un couvert végétal de 30 à 50 centimètres, l'ensemencement et la plantation d'arbres avec la participation de l'ONF (Rapport ICF, 2012). Dans le secteur forestier étudié, l'oubli progressif de l'exploitation minière a été facilité car le paysage «n'est pas marqué par une organisation urbaine reliée à la mine ", les ouvriers logés dans les communes voisines venant pour l'essentiel travailler en vélo (Grasset, 2017, p. 54).

Première séquence de la mise en visibilité, le rapport BRGM-Géodéris 2008 communiqué par la préfecture aux maires des deux communes, évoque la présence de nombreux résidus et déchets miniers subsistant sur le site, en partie stockés dans les galeries, ou en dépôt aérien sous forme d'une digue à stérile sur une surface de 3,5 hectares (ICF, 2012). Puis une étude d'interprétation de l'état des milieux, conduite en 2011 par Stéphane Garcia, conclut que le site pollué de Saint-Félix-de-Pallières n'est pas adapté à un usage résidentiel, en raison « d'un intervalle de risque de 3 pour le scénario d'ingestion de terre " (Garcia, 2011, p. 6). Né de l'impossible remonétarisation du territoire abandonné, le concept de "remise à zéro » et le mythe d'un retour à l'état antérieur ont produit un "espace d'illusion», marqué par une «amnésie volontaire» et l'absence de responsabilité des acteurs (Kohler, 1989, p. 343).

Le risque minier a été minoré voire ignoré par les acteurs politiques locaux, sous forme " d'une négociation silencieuse consistant à détourner le risque " (Gibout et Zwarterook, 2013, p. 139). Quand ce rapport est arrivé en mairie en 2008, les élus l'ont rangé dans le tiroir, déplore les habitants ${ }^{4}$. De son côté, le maire de Saint-Félix-de-Pallières se dédouane de toute responsabilité : «je n'ai pas fait d'étude de chimie, j'étais absolument incapable de savoir jusqu'à quel point c'est pollué», alors même qu'aucune explication n'accompagnait le document (Renon et Jouve, 2016). Dans le courrier joint, le préfet invitait les maires à prendre quelques mesures simples de sécurité, telles que la signalisation des dangers ou la clôture des zones contaminées, préconisations que les élus ont refusées, affirmant ne pas en avoir les moyens techniques et financiers la zone à couvrir étant trop vaste.

Comme on le voit, les élus locaux peu familiarisés aux risques industriels, sont isolés et marginalisés, contrairement aux fonctionnaires de la préfecture et de la Direction régionale de l'industrie, de la recherche et de l'environnement (DRIRE), forts de leurs compétences technico-législatives et de leur légitimité légale-rationnelle, qui « apparaissent volontiers comme les propriétaires du problème, générant chez les élus une attitude assez générale de délégation » (Chabbal, 2005, p. 187).

En Cévennes, comme partout en France "s'ajoute la perception qu'après une brève attention de la Nation, les victimes auraient été laissées seules face à leurs problèmes, aux tergiversations des sociétés minières, aux lenteurs de la justice » (Vaulont et Vo Van Qui, 2011, p. 83), jusqu'à caractériser une discrimination à leur encontre, notamment en regard de l'indemnisation d'autres types de catastrophes. L'argument fait mouche si l'on considère que la commune de Thoiras a été sept fois reconnue en état de catastrophe naturelle entre 1982 et 2008 pour tempête, inondations et coulée de boue, mouvement de terrain, ouvrant rapidement droit à la garantie des assurés.

\section{L'articulation des luttes environnementales et minières}

16 Jouissant d'une tradition d'accueil des huguenots et des maquisards (Bodart, 2009), le territoire cévenol s'est affirmé comme un haut lieu des luttes environnementales, après 
l'annulation de la déclaration d'utilité publique autorisant la construction d'un barrage dans la Vallée Française en mai 1992. Nous faisons l'hypothèse que la vitalité de ces associations locales, forme la résistance et informe la résilience des populations locales évoluant dans des environnements pollués (physiques, climatiques et psychologiques).

\section{Le rôle des associations d'éducation, de défense et protection de l'environnement}

17 Les Cévennes n'ont pas échappé à l'exode rural massif qui a vidé les mas et les hameaux de près de quatre cinquièmes de leurs habitants à partir de 1850 jusqu'au milieu du $\mathrm{XX}^{\mathrm{e}}$ siècle (Clavairolle, 2008). La charte du Parc naturel des Cévennes adoptée en 2007, dépeint ce déclin post-mine contrebalancé dans les années 1970 par l'arrivée des " néo-ruraux » qui ont contribué à une revitalisation des vallées et de la culture cévenole. La déprise frappe l'ensemble du territoire puisqu'au recensement de 1968, il ne subsistait que $30 \%$ de la population de 1850. Les deux communes de notre périmètre d'étude ont subi de plein fouet l'exode urbain puis le renouveau rural, dans les années 1980, comme le montre le graphique 1.

Graphique 1 : Comparaison de l'évolution démographique sur les communes de Thoiras et de SaintFélix-de-Pallières (en centaine d'habitants)

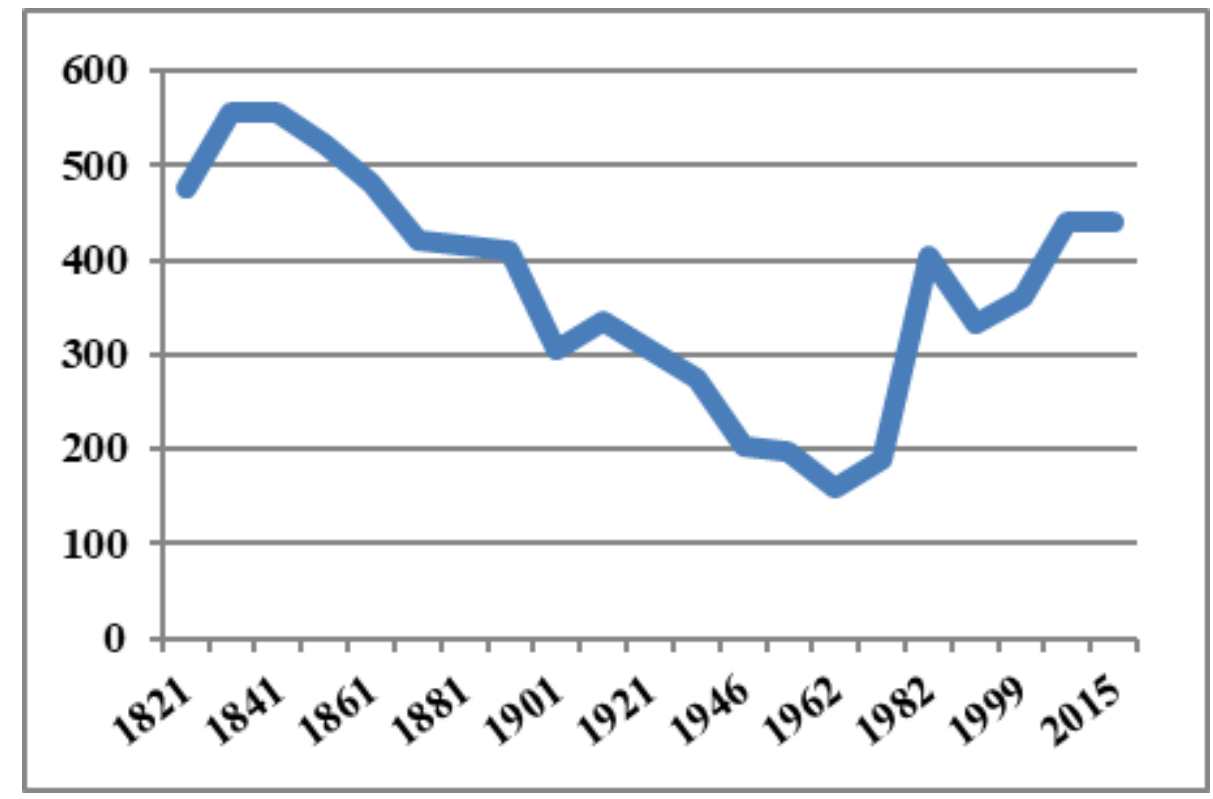

Commune de Thoiras 


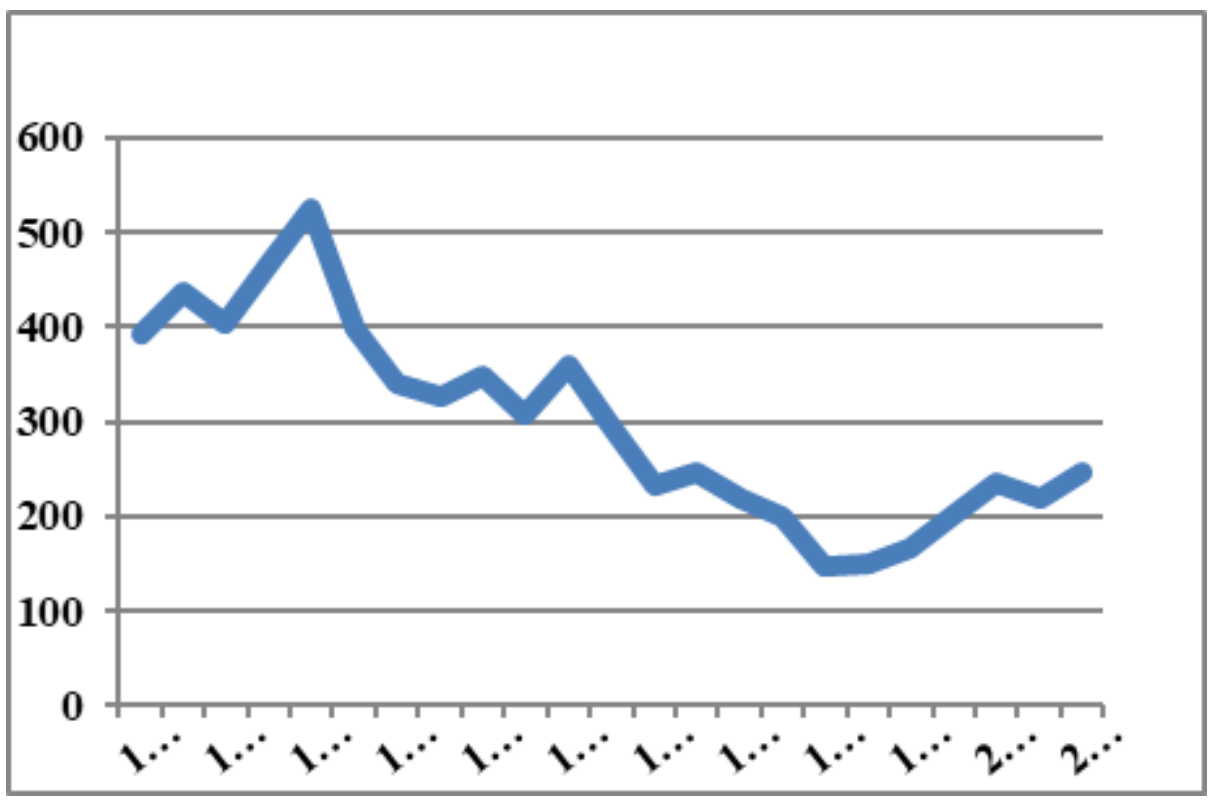

Commune de Saint-Félix-de-Pallières

Source : B. Mésini - Ldh/EHESS/Cassini, puis Insee à partir de 2006

Décrite comme un retour au désert, à la terre et au village (Hervieu et léger, 1979), la première vague d'installation communautaire s'est poursuivie dans les années 1990, conduisant à des occupations durables, par des nouveaux venus aux profils divers, avec un « taux élevé d'individus engagés dans les nouveaux mouvements contestataires » (Clavairolle, 2008, p. 97). Ces mouvements d'éducation populaire ont participé à la construction de l'éducation à l'environnement, dans une logique contestataire de l'écologie politique (Jacqué, 2006).

19 En mettant en relation dans le temps et l'espace des « lieux qui font liens » et des « liens qui font lieux » (Guérin-Pace et Filippova, 2008), les associations illustrent la construction socio-spatiale et culturelle des identités individuelles et collectives. Signe de la reconquête rurale, 33 associations domiciliées sur les communes de Saint-Félix-dePallières et 52 à Thoiras ont été déclarées à la sous-préfecture du Vigan entre 1997 et 2018. La nomenclature des associations déclarées dans la commune de Thoiras depuis 1997 révèle toute l'étendue de leurs activités (Graphique 2). 
Graphique 2 : Nomenclature des 52 associations déclarées sur la commune de Thoiras entre 1997 et 2018

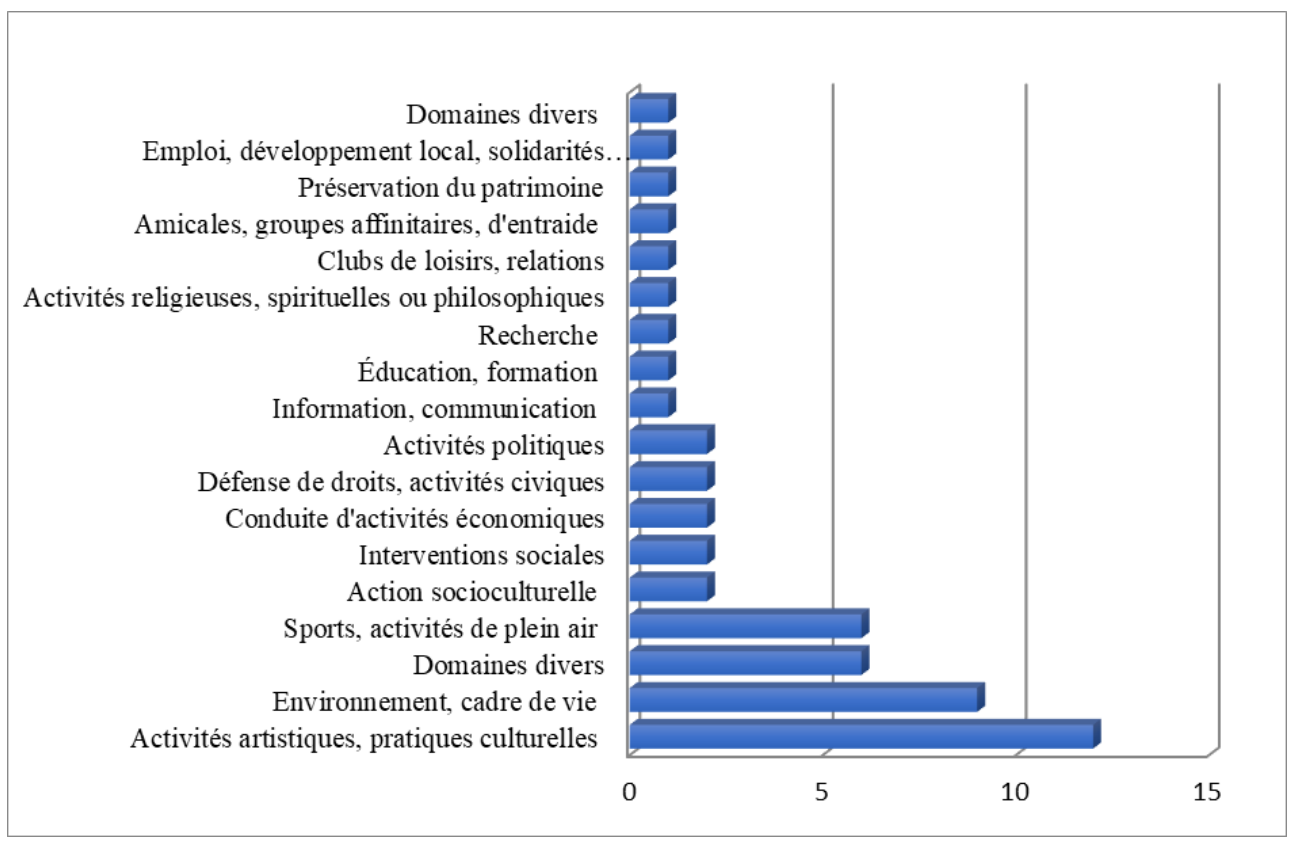

Source : B. Mésini, Bulletin Officiel des associations

Pallières-Gravouillère en raison de l'opportunité d'accès au foncier et du coût modéré des terrains vendus. Le groupement foncier agricole (GFA) La Gravouillère est devenu propriétaire de 30 hectares achetés à la SAFER en 1986, puis de trois autres achetés ultérieurement à la "Sociétê Union minière de France » pour la somme de 14000 francs, en décembre 2000. Ce sont sur ces parcelles que le fils du gérant a créé le 30 août 2003 l'association La Mine, qui développe des activités socio-économiques d'accueil, d'insertion et d'emploi, avec pour mission l'entretien et l'animation du site mais aussi l'accueil et l'accompagnement des personnes en travail d'intérêt général (TIG) envoyées par le parquet de Nîmes. L'association comporte trois catégories de membres (propriétaires, résidents et accueillis). L'espace habité comprend des habitations mobiles ou démontables, des jardins, un espace de formation, un atelier de transformation de bière et de jus de fruits, un poulailler, générant une économie locale sur la base d'accès à des ressources sociales, spatiales et environnementales.

Également déclarée le 3 mars 2008 sur le site, l'association Le Filon a pour objectif de « permettre l'acquisition de savoirs techniques par l'éducation populaire en développant des actions pédagogiques, des cours et des journées auprès de tout public (scolaires ou adultes) sur les pratiques respectueuses l'environnement $»^{5}$. Enfin, à visée écologiste et ouverte à de nombreux autres collectifs locaux et nationaux, l'Association des Rencontres des Objecteurs de Croissance a été créée en 2008 par le propriétaire du GFA Gravouillère, à des fins d'information, de mobilisation, d'action et de prévention. Issue de l'éducation populaire et de la prévoyance, elle se fixe pour objectif de "protéger la nature, la biodiversité sauvage ou domestique, l'homme et son environnement de toutes les nuisances et pollution, d'informer sur les impacts de tout ordre (écologique, sanitaire, social et économique) mais aussi d'assister ou de soutenir les collectifs locaux agissant dans les mêmes domaines $"^{6}$. 
Comme l'illustre le graphique 3 , le thème de l'environnement s'avère central dans les activités des dix associations domiciliées sur Pallières.

Graphique $n^{\circ} 3$ : Nuage de mots à partir des objets de dix associations déclarées sur le hameau de Pallières

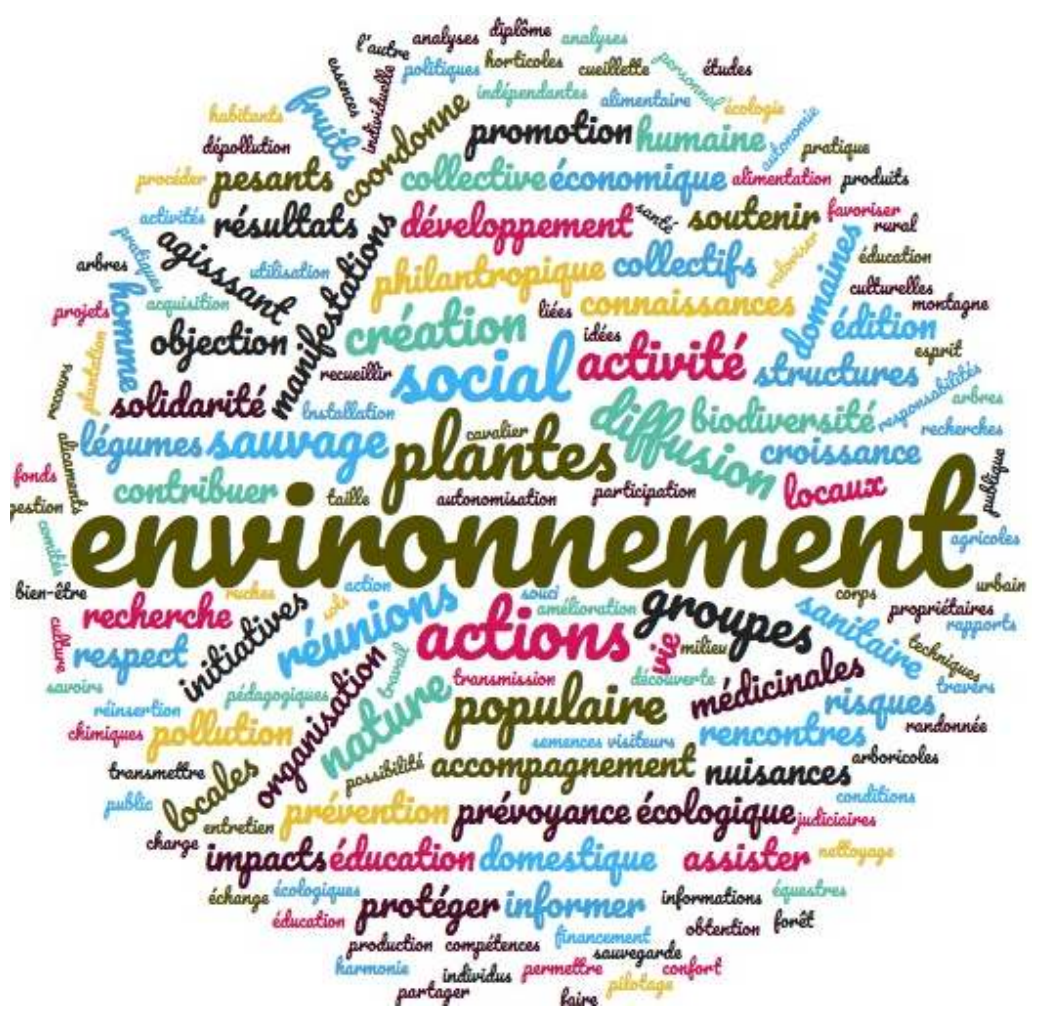

Source : B. Mésini 
collectifs anti-mines ont organisé un festival à Couflens en août 2018, pour lutter contre un permis d'exploration de tungstène et d'or accordé à une société australienne.

\section{Une épidémiologie critique}

Lorsqu'en 1986, messieurs S., B. et P. ont acquis 30 hectares de terrains remembrés pour créer le GFA dont la production est cultivée en label bio, nulle part n'est fait mention de la pollution des sols. Situés en zone agricole, les terrains vendus par la SAFER comportaient deux ruines, dont le POS prévoyait la possible restauration. Les habitats légers et mobiles des résidents, accueillis par l'espace d'accueil de la Mine, sont eux stationnés sur les emplacements des anciens bâtiments d'exploitation de la mine, au lieu-dit « La Cantine » sur la commune de Saint-Félix-de-Pallières, et au lieu-dit "Gravouillère », au niveau des Puits $n^{\circ} 3$ et 3 bis, face à la digue à stérile sur la commune de Thoiras.

Dans un second temps, c'est l'industriel Umicore qui a contacté les propriétaires GFA pour proposer la vente de deux autres parcelles. Ce deuxième acte notarié évoque le potentiel toxique puisque subsiste une servitude sur le terril, "mais nulle part n'était mentionné le risque effondrement $»^{11}$. Or, le vendeur d'un terrain, sur le tréfonds duquel une mine a été exploitée, est tenu d'informer par écrit l'acheteur des dangers ou inconvénients importants qui résultent de l'exploitation, obligation introduite dans le code minier par la loi du 14 juillet 1994. Pourtant, dans le titre de propriété daté du 26 janvier 2001, les biens désignés «ne font l'objet d'aucune interdiction d'habiter, ni d'un arrêté de péril $»^{12}$.

\section{Photo 1 : Espace d'accueil la Mine}

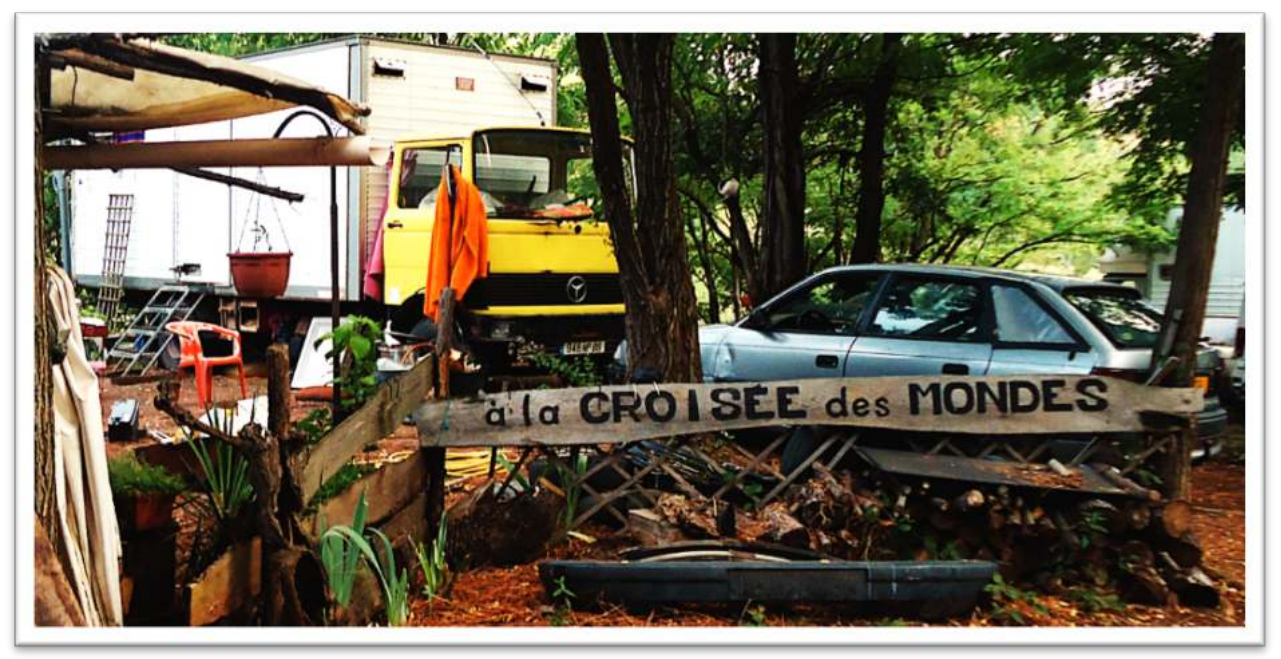

Crédit - Béatrice Mésini, 2016 


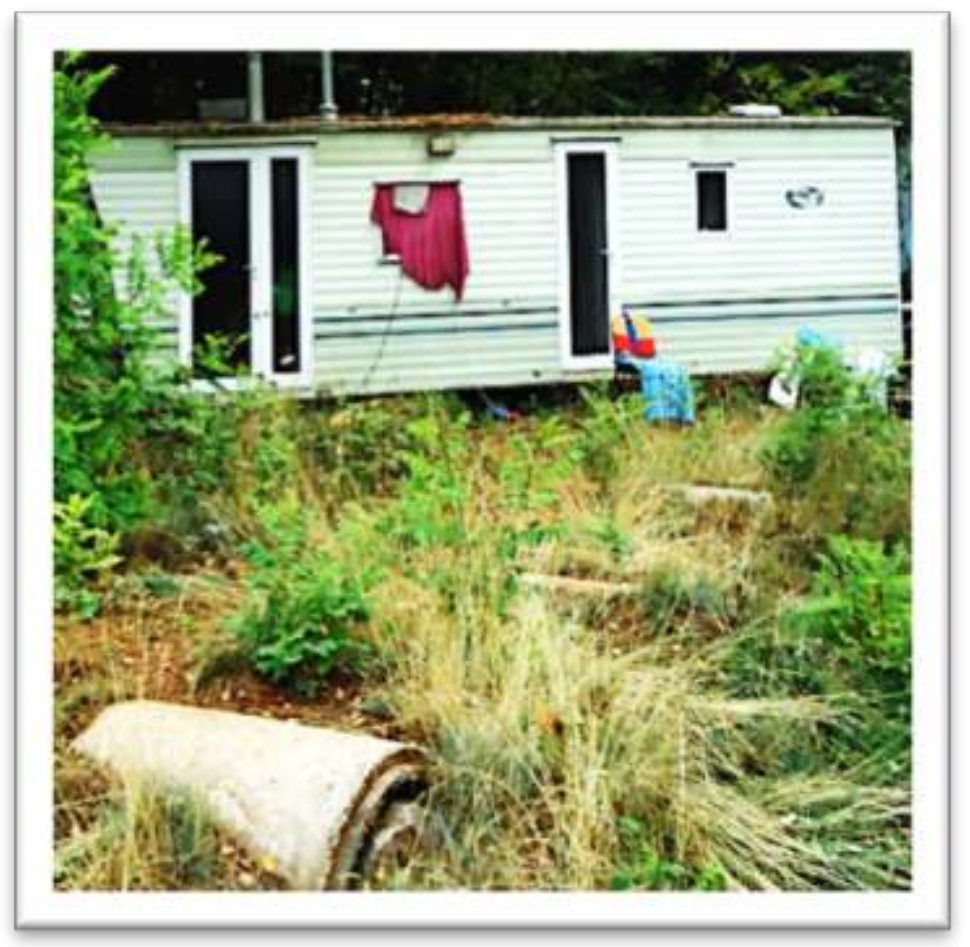

Crédit - Béatrice Mésini, 2016

Photo 3 : Fabrique artisanale de bière

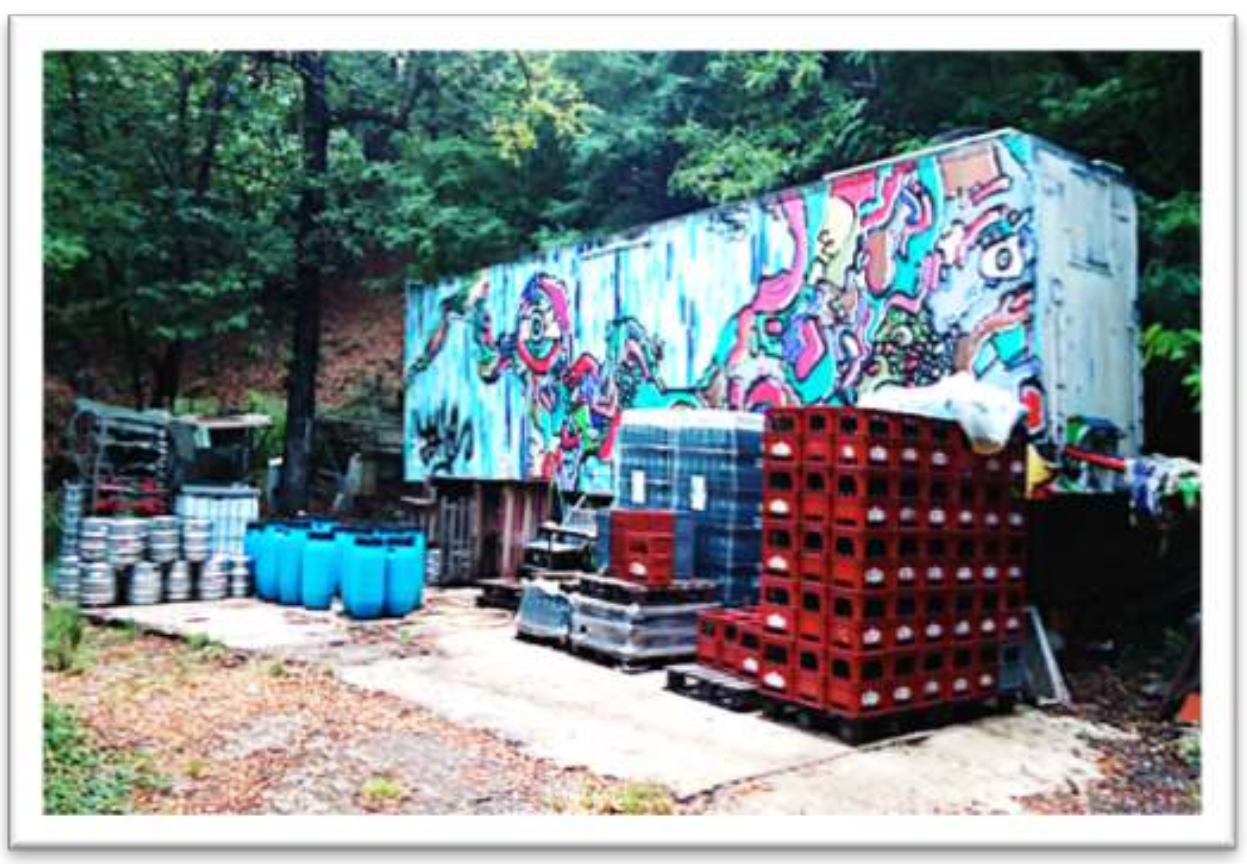

Crédit - Béatrice Mésini, 2016

Le silence a perduré pour bien d'autres acheteurs : un couple a obtenu un permis de construire délivré en 2006 par la mairie qui les a rassuré en «présentant le chemin de 
Pallières comme un pôle d'attraction pour les chasseurs, amateurs de préhistoire et randonneurs " ${ }^{13}$. Quant aux derniers installés, jeunes agriculteurs, artisans ou retraités rencontrés sur le site en août 2016, ils n'ont pas été informés de la pollution résiduelle. La méconnaissance des aléas et risques miniers relèvent de l'amnésie d'un territoire reconverti en espace touristique et résidentiel : «la digue, tout le monde y jouait, c'était notre terrain de jeux et celui de tous les enfants de la vallée » témoigne le fils du gérant du GFA ${ }^{14}$, notamment pour tous les adeptes de vélos, motos et de quads.

La direction régionale de l'environnement, de l'aménagement et du logement (DREAL) du Languedoc-Roussillon a lancé des investigations complémentaires concernant les habitations du hameau de Pallières en $2015^{15}$. Pour autant, les prélèvements effectués ont été formellement récusés par l'association des riverains des mines de Croix-de-Pallières, « car seuls 150 à 200 l'ont été sur les 900 prévus, étalonnés par un appareil d'analyses de surface ne pouvant pas être utilisé en matière de procédure judiciaire $»^{16}$. Face aux doutes, l'association a lancé deux financements participatifs pour réaliser une analyse indépendante sur la qualité de l'eau, les militants estimant que les prélèvements n'avaient pas été faits aux bonnes périodes, ni aux bons endroits.

Définie commela production par les "profanes" de connaissances sur les risques environnementaux et technologiques, l'épidémiologie populaire renvoie à des processus plus larges par la manière dont ils rassemblent les données, collaborent avec des experts, en recrutent d'autres, mettent sur pied une organisation communautaire et participent aux enquêtes épidémiologiques, sans idée parfois de l'ampleur de leur implication future (Brown, 2010). De leur côté, les habitants du hameau de Pallières ont approuvé la mise en place de l'étude épidémiologique, qu'ils sont quatre à avoir acceptée car ils «mangeaient la production de leurs jardins et basse-cour ${ }^{17}$. Toutefois, ils critiquent la non prise en compte par les études toxicologiques des processus de bioaccumulation, de bioconcentration et de bioamplification (Camizuli, 2013), ainsi que le choix des traceurs retenus : « Les analyses des traceurs n'ont porté que sur trois polluants, sachant qu'il faut compter 35 à 70 euros l'analyse selon les produits recherchés. Ce choix d'ordre financier est problématique, car d'autres polluants s'avèrent plus dangereux, à l'instar du thallium ${ }^{18}$. Les habitants questionnent et contestent les hypothèses, les valeurs et la construction sociale du savoir scientifique.

Quand le préfet et l'ARS ont entrepris de diffuser individuellement les résultats des analyses en raison du secret médical, les habitants de la Mine ont contacté les riverains et collecté les résultats pour faire leur propre tableau récapitulatif: «on s'est alors rendu compte que le secret recouvrait tout, car à la préfecture, ils étaient certains que les 46 malades venaient seulement de chez nous, alors qu'ils ne sont que trois ${ }^{19}$. Ils considèrent qu'ils ont mis le doigt «sur les failles de l'État, car on a réussi à ouvrir le débat à toute la zone polluée et pas uniquement sur notre situation ici. Les riverains ont découvert que c'était pollué partout, avec des gros pics de concentration dans et hors l'ancien site minier ».

Dotés de multiples capitaux militants (écologistes, décroissants ${ }^{20}$ et altermondialistes), les habitants de Pallières ont signalé à Umicore la pollution rémanente, puis ont effectué leurs propres recherches, pour s'informer de ses effets auprès de l'École des mines d'Alès. À présent, ils souhaitent parvenir à " présenter un projet de confinement des pollutions et de phyto-remédiation aux autorités et aux scientifiques, avec une croûte étanche d'argile, du gravier pour drainer, de la terre végétale et des végétaux $»^{21}$. À travers le concept d'épidémiologie critique, Brown décrit cette démarche qui consiste à questionner la neutralité supposée d'un modèle, à accorder un grand intérêt au contexte social dans lequel se manifestent les 
maladies et les risques environnementaux, à remédier aux problèmes existants et à prévenir l'émergence de situations de risques et de maladies (Brown, 2010, p. 47).

Dénonçant l'inaction des pouvoirs publics face aux 2 millions de tonnes de déchets et 600000 autres tonnes de stériles de mines, quarante-trois personnes et les deux associations ADAMVM et Générations Futures ${ }^{22}$, ont porté plainte contre $\mathrm{X}$ auprès du tribunal d'Alès et auprès du pôle santé du tribunal de grande instance de Marseille en mars 2016, pour « mise en danger de la vie d'autrui, blessures involontaires et pollution des eaux ». Le budget consacré à l'action juridique est conséquent : en frais d'avocat (3 000 euros), analyses en laboratoire (4 100 euros) et frais d'experts judiciaires (3 334 euros) ${ }^{23}$. D'autres plaintes visent des faits « d'atteinte involontaire à l'intégrité physique » et de " tromperie sur la marchandise entraînant un danger pour la santé de l'homme et l'animal $»^{24}$. En effet, les coûts ne sont pas à la portée de toutes les associations, car la charge de la preuve de la causalité d'une atteinte à l'environnement est délicate pour des raisons de technicité et de prix (Aguila, 2012). Comme on le voit, les mobilisations sanitaires sont coextensives aux causes environnementales, c'est-à-dire capables de s'étendre et s'entendre sur des préceptes de prévention, de réparation et de remédiation face aux périls, dans une optique de résilience des milieux socio-naturels.

\section{Quelle gouvernance de l'après-mine, du local au global?}

À quelle échelle interministérielle s'exerce la gouvernance, s'interroge le député Olivier Gaillard qui, après avoir rencontré les maires de Saint-Félix-de-Pallières, Thoiras et Tornac a sollicité les trois ministères de la Transition écologique, de la Santé et des Solidarités et de l'Intérieur. Le premier ministre lui a personnellement répondu que les leçons tirées de l'expérience du Gard faisaient l'objet d'un examen attentif en vue de la réforme annoncée du code minier en $2019^{25}$.

\section{La confiscation de l'information et le confinement des risques}

Administrer les risques est une opération délicate qui revient à " produire une signification socialement lisible à des compromis d'intérêts instables, fondés sur des assemblages et des mises en cohérence d'informations disparates, voire antagonistes en les formulant en termes de prévention et de gestion des risques » (Lascoumes, 1994, p. 153).

Dans notre cas d'étude, l'insuffisance du porter à connaissance est unanimement décriée par habitants et riverains des sites qui dénoncent l'absence de signalétique à destination des usagers dans ce secteur forestier fréquenté par des cueilleurs, chasseurs, amateurs de cross et randonneurs, partie intégrante d'un circuit touristique de visite des dolmens. À cet effet, le dossier départemental des risques majeurs établi en 2005 par le préfet du Gard et actualisé en 2013, renvoie à la responsabilité personnelle des citoyens qui « doivent entreprendre une véritable démarche personnelle, visant à s'informer sur les risques qui les menacent individuellement et sur les mesures à adopter en fonction de sa propre vulnérabilité, celle de son environnement (habitat, milieu, etc.) et des dispositions possibles pour la minimiser " (Dossier départemental des risques majeurs du Gard, p. 4).

37 Le médecin en charge de ce dossier pour le compte de l'agence régionale de santé estime que la situation est mal maîtrisée, en raison de la contradiction de plusieurs logiques 
antagonistes: "si nous informons les populations, nous créons de l'angoisse, nous mettons en cause l'image du territoire et par voie de conséquence la valeur des biens ». C'est ce qui explique la «focalisation des riverains sur les festivals et les manifestations estivales à la Mine, on assiste à un transfert, laissant entendre qu'il est plus facile de s'en prendre à ces nuisances qu'aux véritables questions de santé publique » (Picot, et al., 2013, p. 30-31).

Organisateur d'une réunion publique le 9 décembre 2014 à Thoiras, le sous-préfet du Vigan a annoncé aux citoyens, habitants et militants, incrédules et excédés, qu'il n'y aurait pas d'étude épidémiologique, ni de mesures de dépollution : «Si vous pensez qu'on va engager ici des mesures de dépollution, la réponse est non. L'État va-t-il mener une étude épidémiologique? L'ARS refuse car il n'y a pas assez d'habitants, dans des petites communes et pas de statistiques des victimes ${ }^{26}$. Néanmoins, suite à l'inquiétude grandissante des populations locales, deux études de dépistage et d'analyse épidémiologique ont été conduites par l'ARS avec l'appui de la Cellule interrégionale d'épidémiologie, « dans le but de contribuer à rassurer les populations par une démarche de transparence » et « afin de disposer d'éléments de nature à permettre d'éclairer les liens de corrélation et de causalité entre exposition et imprégnation » (Rapport Delaunay, Tardivon, Besson, 2016, p. 21).

Afin de rétablir de la confiance dans les échanges, la mise en place d'un premier comité de suivi et d'information a réuni les parlementaires, les maires, les responsables associatifs et le vice-président d'Umicore le 7 juin 2016. Interrogé sur le danger sanitaire encouru par les populations, le vice-président conteste la preuve de la causalité en l'absence d'étude officielle corrélant la santé et l'exploitation minière : «seules les autorités sanitaires ont la légitimité nécessaire, la compétence scientifique, la rigueur et le sérieux nécessaires pour mener à bien des études épidémiologiques ${ }^{27}$. Comme naguère, les liens entre l'état du milieu et l'état de santé se sont triplement défaits, en s'effaçant devant une médecine privée et libérale et devant les ingénieurs ayant établi leur juridiction sur les pollutions avec leurs propres outils (Kalaora et Vlassopoulos, 2013), parfois en étroite coopération avec le gouvernement et le monde industriel (Chastagnaret, 2017).

Conduite à partir d'octobre 2015, l'étude sanitaire a porté sur 651 personnes volontaires, dont 564 personnes de plus de 15 ans et 87 enfants de moins de 15 ans, suivie d'une deuxième phase de prélèvements auprès de 894 personnes en février 2016. Les premiers résultats indiquent que $22 \%$ présentent une imprégnation à l'arsenic supérieure à la valeur de référence établie en population générale, et $13 \%$ présentaient une imprégnation au cadmium supérieure à la valeur de référence établie en population générale. Pour autant la causalité entre l'exposition et les pathologies n'est pas prouvée écrit la directrice générale de l'Agence régionale de santé d'Occitanie au médecin qui préside l'association ADAMVM, en avril 2018: «Une étude d'épidémiologie comparant l'incidence de pathologies autour d'un site pollué avec l'incidence observée dans une zone de référence, ne permet pas à elle-seule de juger de la causalité entre l'exposition et la maladie étudiée. Même si le lien entre la pathologie observée et le polluant étudié est connu, les pathologies sont souvent d'origine multifactorielle. À ce jour, il n'existe aucun marqueur biologique permettant de relier un type histologique de cancer donné et une exposition environnementale ${ }^{28}$.

41 Le 2 juillet 2018, le préfet du Gard a engagé une procédure de mise en demeure contre l'entreprise Umicore pour l'obliger à confiner les déchets laissés sur cinq anciens sites miniers du Gard, dossier qui devrait faire selon lui « jurisprudence » dans un contexte de no man's land juridique ${ }^{29}$. Le préfet a pris son arrêté estimant que les anciens exploitants restent détenteurs des déchets produits, alors que ces derniers s'estiment relevés de leurs obligations puisqu'ils ont quitté le site depuis cinquante ans. En attendant, les cinq sites 
considérés comme dangereux devront être clôturés : trois sur la commune de Saint-Félixde-Pallières (la mine Joseph, le dépôt circulaire de l'Issart, et le puits $n^{\circ} 1$ ) et deux sur celle de Thoiras (les haldes du GFA de la Gravouillère et la digue ou dépôt).

\section{Un cumul d'inégalité, d'injustice et de discrimination}

42 Avant d'être reconnus et admis dans leur statut de victimes, les résidents de la mine ont d'abord été présentés comme responsables au premier chef des nuisances et risques engendrés par leurs pratiques, dont ils ont dû supporter la charge sociale, médiatique et politique. Lors d'une visite effectuée en juin 2007, le responsable de l'Unité Risques Géotechniques et l'ingénieur de l'Unité Risques Naturels, Ouvrages et Stockages de l'INERIS mentionnent plusieurs désordres apparents, notant que la « plate-forme sommitale des haldes est occupée par une population de marginaux (néo-ruraux), qui stockent un ensemble hétéroclite de matériel (nombreux véhicules divers, récupération...) et logent sur place» (INERIS, 2009, p. 9). Intimement liées à l'ordre public, les notions de propreté et de tranquillité questionnent la «double acception des déchets» naturels ou sociaux, qui doivent être limités, nettoyés et éloignés (Guienne, 2006, p. 37). Le premier combat des habitants du hameau de Pallières a donc consisté à lutter contre le dénigrement social, entendu comme le fait de "juger négativement la valeur sociale de certains individus ou de certains groupes, en déconsidérant leur mode de vie » (Honneth, 1992, p.164).

Les inégalités environnementales peuvent être discriminatoires, quand «l'exposition de certains groupes sociaux est jugée disproportionnée en regard de celles qui affectent d'autres catégories sociales" (Larrère, 2017, p. 7). C'est une situation de marginalité sociale que dépeint le maire d'Anduze, pour qui la vérité est à la fois crue et cruelle : "S'il n'y avait pas la problématique sociale d'occupation de l'espace par des gens qui ne faisaient pas partie de notre paysage, on continuerait à manger nos champignons". L'appréciation est complétée par le maire de Thoiras: "Si le site avait été remis à la nature avec des chèvres et des moutons, personne n'aurait rien remarqué parce que c'était du camouflage, mais toutes ces manifestations qui se sont greffées sur le carreau de la mine ont dévoilé les risques de pollution " (Renon et Jouve, 2014) ${ }^{30}$. Pourtant, si l'on considère la chaîne des responsabilités, c'est en amont des installations qu'il incombait aux élus de rendre la zone polluée non constructible, au nom du risque encouru (Laurent, 2017).

À ce jour, la situation des résidents du hameau de Pallières est inextricable : la procédure d'insalubrité est inadéquate "car la cause de la pollution n'est pas imputable aux propriétaires et que les travaux sur le bâti ne suffiraient pas à remédier à la pollution $»^{31}$. Les gérants sont poursuivis en correctionnel pour installation d'une aire d'accueil de caravanes et d'habitations légères de loisirs, d'une aire de stationnement de camions et d'un dépôt de véhicules, en violation du règlement de la zone ND du POS de la commune. Par décision rendue en septembre 2018, le tribunal correctionnel d'Alès a condamné le GFA, l'association la Mine et leurs deux dirigeants au paiement de 1000 euros chacun, ainsi qu'à l'enlèvement de 14 véhicules, d'un semi-remorque, de trois caravanes et des décors, dans un délai de 6 mois et sous astreinte de 50 euros par jour de retard. Ainsi privés de leurs résidences principales et de leurs moyens d'existence, les habitants ont décidé de pourvoir en appel.

Une deuxième injustice consisterait à traiter différemment dans l'indemnisation les propriétaires clausés et non clausés. Les risques de mouvements de terrain et d'effondrement sur les sites miniers étant parfaitement connus des industriels, certains 
vendeurs ont pris la précaution d'insérer dans les contrats de vente une "clause minière » les exonérant de toute responsabilitê en cas de désordres, sans parfois que «le nouvel acquéreur n'ait pleinement conscience de sa signification " (Vaulont, Vo Van Qui, 2011, p. 79). Lorsqu'une telle clause a été valablement insérée dans un contrat de mutation immobilière, l'État assure dans les meilleurs délais l'indemnisation des dommages résultant du sinistre minier. Après les effondrements d'Auboué et de Moutiers dans le bassin ferrifère lorrain, les premiers sinistrés clausés ont été indemnisés, sur la base d'accords amiables passés entre l'État, l'ancien exploitant minier et son assurance (Duchêne, 2008). Pourtant, les biens immobiliers clausés et les biens meubles habités ${ }^{32}$ du hameau de Pallières, risquent fort d'être exclus de toute indemnisation.

Un puissant sentiment d'injustice relève aussi de la dépréciation de la valeur des biens immobiliers dans les secteurs pollués. Outre les communes étudiées d'autres sont aussi affectées, comme celles d'Anduze, de Durfort, de Générargues... : « Ni les notaires, ni les agences ne veulent prendre les maisons en vente, quant aux assureurs, il ne se déplacent même plus dans le périmètre " ${ }^{33}$. Il est à craindre que le phénomène s'amplifie car le Gard est un département très marqué par le risque minier, 104 communes sont touchées par un aléa dans le périmètre départemental (DDRM, 2013). Au demeurant, quelle évaluation faire des immeubles : sur la base d'une reconstruction neuve équivalente, ou sur la valeur vénale du bien avant les dommages? L'indemnisation des dommages consiste en la remise en l'état de l'immeuble sinistré, mais lorsque l'ampleur des dégâts subis la rend impossible, l'indemnisation doit permettre au propriétaire de l'immeuble sinistré de " recouvrer dans les meilleurs délais la propriété d'un immeuble de consistance et de confort équivalents ${ }^{34}$.

Un dernier déni de justice concerne de facto tous les plaignants, surtout ceux qui sont privés de leur habitation principale, en raison de la lenteur des procédures, «certains dossiers courent toujours depuis plus de 15 ans, selon des délais insupportables pour la plupart des particuliers » (Vaulont, Vo Van Qui, 2011, p. 70). De plus parce qu'ils sont dénués de tout caractère matériel, les troubles liés aux conditions d'existence, le préjudice moral et les atteintes résultant des répercussions du sinistre sur la santé physique ou mentale, ne sont pas indemnisables ${ }^{35}$, alors même que ce sont les plus préjudiciables.

\section{Conclusion}

La question des atteintes à l'environnement touche à la survie des populations dès lors qu'elle « recouvre la destruction des conditions écologiques à partir desquelles s'est développé un mode de vie spécifique ». Comme nous y convie Lafargue, il y a une nécessité d'élargir les formes d'atteintes à l'environnement pour y inclure le préjudice civilisationnel et " envisager non plus un droit de l'environnement, mais un véritable droit du "milieu naturel" " (Lafargue, 2010, p. 156 et 159).

La justice environnementale suppose le droit à l'information et à la participation des citoyens aux politiques publiques, concernant la protection de l'environnement. Nous avons vu qu'à travers l'action des associations mobilisées, tant sur le plan local que national, se développait un processus deformation à une citoyenneté sociale et écologique qui contribue à l'émergence d'une responsabilité partagée, sociale et environnementale " c'est-à-dire à l'exercice continu et interrelié des droits et des responsabilités "(Orellana, Marleau, 2015, p.10). De ce point de vue, la constitution d'un capital 
individuel (cognitif) et collectif (interactif), pourrait agir comme facteurs de réduction de l'incertitude et de résilience.

Pour autant, comment partager «le fardeau des déchets » au cours du temps entre les sphères publique et privée, s'interroge Thomas Schellenberger. Car les sols sont à la fois des milieux vivants et des supports de prérogatives en droit de l'urbanisme et de l'environnement (Thomas Schellenberger, 2014). Après l'arrêt de l'exploitation, la renonciation et la vente des terres exploitées, qui sont les producteurs, détenteurs et responsables des déchets sur le sol et dans le sous-sol, des toxiques dispersés dans l'environnement et des sinistres miniers?

La réforme du code minier se fait attendre depuis six ans. Le député socialiste, écologiste et républicain de l'Indre justifie l'actuelle réforme de ce code qui ne répond plus ni aux attentes sociales, ni environnementales, qui est devenu une source de complexité et d'imprévisibilité pour les opérateurs (Chanteguet, 2017). En prévoyant l'intervention du fonds de garantie devant des situations imprévisibles et imprévues, où la bonne foi et l'honnêteté des exploitants ne sont pas en cause, la « solidarité nationale » permettra-telle de concilier les intérêts de chacune des parties dans la gestion de l'après mine ? Rien n'est moins sûr car ce sont parfois des intérêts divergents et concurrents qui s'énoncent tout autant dans la mise en sécurité des populations, la qualification des dommages, la répartition des responsabilités, que dans l'anticipation de risques futurs (fracturation hydraulique) ou d'opportunités nouvelles pour les exploitants à travers le réemploi des résidus miniers.

Par leur gestion agri-environnementale des sols, les habitants du hameau de Pallières se prévalent de la mise en œuvre du précepte «d'utilisation durable» du territoire, selon lequel «la pratique des usages peut être un instrument qui contribue à la biodiversité, par l'utilisation des meilleures techniques disponibles à un coût économiquement acceptable ». En contrepoint, quelle application feront les acteurs économiques, politiques et judiciaires des principes « d'action préventive et de correction, par priorité à la source, des atteintes à l'environnement ", de " pollueur-payeur » et de "solidarité écologique ", qui appellent " toute prise de décision publique ayant une incidence notable sur l'environnement à prendre en compte les interactions des écosystèmes, des êtres vivants et des milieux naturels ou aménagés $»^{36}$ ?

\section{BIBLIOGRAPHIE}

\section{ARTICLES ET OUVRAGES}

AGUILA, Y., 2012, Dix propositions pour mieux réparer le dommage environnemental, Environnement, $\mathrm{n}^{\circ}$ 7, dossier 2, juillet 2012, p. 1-8.

BODART M., 2009, Territoire de renaissance, territoire de création. L'étude des porteurs de projet néo ruraux en Ardèche méridionale, Mémoire de Master 1, mention sciences sociales, ENS, Lyon, 31 p. 
BRETESCHE S et PONNET M., 2013, Le risque environnemental entre oubli et gestion du passé : le cas d'une ancienne mine d'uranium française, Annales des Mines - Gérer et comprendre, ${ }^{\circ} 111$, p. 15-24.

BROWN P., 2010, Retour sur l'épidémiologie populaire, Presses des Mines, p. 53-87.

CAMIZULI E., 2013, Impact des anciens sites miniers et métallurgiques sur des écosystèmes terrestre et aquatique actuels. Étude comparative de deux moyennes montagnes : le Morvan et les Cévennes, Thèse d'archéologie sous la direction de Fabrice Monna, Vol.1, soutenue le 13 décembre, Université de Bourgogne, $228 \mathrm{p}$.

CHABBAL J., 2005, Le risque invisible. La non-émergence d'un problème public, Politix, De Boeck Supérieur, nº 70, p. 169 à 195.

CHARLES N., DUPUY J.-J., CHRISTMANN P., GALIN R., GUILLON D., 2017, Industrie minérale et activité minière en France, collection La mine en France, tome 1, 25 p.

CHRISTEN G. et HAMMAN P., 2015, Transition énergétique et inégalités environnementales, énergies renouvelables et implications citoyennes en Alsace, Strasbourg, Presses Universitaires de Strasbourg, $228 \mathrm{p}$.

CHASTAGNARET G., 2017, De fumées et de sang. Pollution minière et massacre de masse. AndalousieXIXème siècle, Madrid, Bibliothèque de la Casa de Velázquez, 456 p.

DUCHÊNE F., 2008, Les affaissements miniers dans le bassin ferrifère lorrain : quand le territoire re-politise la gestion du risque, Développement durable et territoires [En ligne], Dossier 11 : Catastrophes et Territoires, mis en ligne le 30 octobre 2008. URL : http:// developpementdurable.revues.org/7073.

FONTAINE M., 2016, Visible/invisible : Ce qui reste des mines, Techniques \& Culture, $n^{\circ}$ 65-66, p. 74-91.

GIBOUT C., ZWARTEROOK I., 2013, Gérer les risques industriels et la pollution dans le Dunkerquois : une double échelle transactionnelle, De Boeck Supérieur, « Pensée plurielle », $\mathrm{n}^{\circ}$ 33-34, p. 131 à 148.

GUIENNE V., 2006, L'injustice sociale. L'action publique en questions, Erès, collection sociologie clinique, $190 \mathrm{p}$.

GUÉRIN-PACE F. ET FILIPPOVA E. 2008, Ces lieux qui nous habitent. Identité des territoires, territoires des identités, Paris, L'Aube, $276 \mathrm{p}$.

HERVIEU-LEGER D. et HERVIEU B., 1979, Le retour à la nature : au fond de la forêt, l'État. Paris, Éditions du Seuil, 240 p.

HONNETH A., 1992, La lutte pour la reconnaissance, Paris, Édition du Cerf, 232 p.

JACQUÉ, M., 2016, L'éducation à l'environnement : entre engagements utopistes et intégration idéologique, Cahiers de l'action, vol. 47, n¹, p. 13-19.

KALAORA B., VLASSOPOULOS C., 2013, Pour une sociologie de l'environnement. Environnement, société et politique, Champ Vallon, $320 \mathrm{p}$.

KEHLER D., 1989, Essai d'analyse comparative des politiques de traitement et de reconversion des friches sidérurgiques dans les régions Nord - Pas-de-Calais et Ruhr, Hommes et Terres du Nord, $\mathrm{n}^{\circ} 4$, p. 336-345.

LAFARGUE R., 2010, Le préjudice civilisationnel pour atteinte à l'environnement. Droit au cadre naturel et réalités socio-culturelles : interdépendances et interdisciplinarité, Éditions juridiques associées, Droit et société, $\mathrm{n}^{\circ} 74$, p. 151 à 169. 
LARRERE C., 2017, Les inégalités environnementales, Paris, PUF, collection « La vie des idées », 99 p.

LAURENT E., 2017, Mesurer et réduire les inégalités environnementales en France, in LARRERE C., Les inégalités environnementales, Paris, PUF, collection « La vie des idées », p. 29-51.

LASCOUMES P., 1994, L'éco-pouvoir. Environnement et politiques, Paris, La Découverte.

LE BLANC A., FRÈRE S., HELLEQUIN A.P., FLANQUART H., GONTHIER F. et CALVO-MENDIETA I., 2009, Le jeu de la concertation autour des sites Seveso : Une analyse des dispositifs de gouvernance locale dans l'agglomération dunkerquoise, Vertigo, hors-série 6, ACFAS, p. 1-6.

ORELLANA I. ET MARLEAU M.-È., 2015, Résistance, engagement et construction d'une écocitoyenneté critique, in SAUVÉ L. et VAN STEEBERGHE É., Identités et engagements : enjeux pour l'éducation relative à l'environnement, Éducation relative à l'environnement : Regards - Recherches Réflexions, volume 12, p. 59-80.

PAQUOT T., 2005, Habitat, habitation, habiter. Ce que parler veut dire..., Informations sociales, $\mathrm{n}^{\circ}$ 123, p. $48-54$.

REGHEZZA-ZITT M., RIGAUD É., SANSEVERINO-GODFRIN V., 2012, Aménagement durable des territoires soumis à de fortes contraintes environnementales : enjeux et perspectives. Exemple de la vallée du Var (06) », in MESINI B., Aménagement durable des territoires ruraux, Nice, PUAM, p. $175-187$.

SCHELLENBERGER T. 2014, Le droit public des utilisations du sous-sol - Réflexions sur le régime juridique des stockages géologiques de déchets, thèse soutenue sous la direction de LAMBERT M.-L., Faculté de droit d'Aix-Marseille Université.

STEINMETZ B., 2008, Préjudice écologique et réparation des atteintes à l'environnement. Plaidoyer pour une catégorie nouvelle de préjudice, Revue Européenne de Droit de l'Environnement, vol. $12, \mathrm{n}^{\circ} 4$, p. 407-419.

\section{DOSSIERS ET RAPPORTS}

CHANTEGUET J.-P., 2017, rapport portant adaptation du code minier au droit de l'environnement ( $\left.n^{\circ} 4251\right)$, enregistré à la Présidence de l'Assemblée nationale le 18 janvier.

Dossier Départemental des Risques Majeurs du Gard, département du Gard, 2013, 99 p.

GARCIA S., 2011, Interprétation de l'État des Milieux-Anciennes mines de Pallières, (Gard), 15 p.

GOSSEMENT A., 2011, Droit minier et droit de l'environnement. Éléments de réflexion pour une reforme relative à l'évaluation environnementale, à l'information et à la participation du public, Rapport remis à la Ministre de l'Écologie, du Développement durable, des Transports et du Logement, $372 \mathrm{p}$.

GRASSET A., 2017, Les mines de la Croix de Pallieres. L'histoire des mines et leur impact socio-économique sur le territoire de Tornac et ses alentours, Mémoire dans le cadre d'un service civique à la mairie de Tornac, 125 pages.

ICF ENVIRONNEMENT, 2012, Diagnostic environnemental de l'interprétation de l'état des milieux des anciens sites miniers sur les communes de Saint-Félix-de-Pallières et Thoiras, rapport Phase 1 «Étude historique et document recensement des usages », Aix-en-Provence, 128 p.

ICF ENVIRONNEMENT-BRGM, 2013, Diagnostic environnemental. Interprétation de l'état des milieux. Anciens sites miniers sur les communes de Saint-Felix-de-Pallières et Thoiras (30), AIX/12/085IR -VP2, 76 p.

INERIS, 2007, Audit de la digue de résidus de traitement du gisement de Saint-Félix-de-Pallières (30), rapport d'études DRS-07-90078-11924A. 
LE DÉAUT J.-Y., 1999, Rapport sur la proposition de loi relative à la responsabilité en matière de dommages consécutifs à l'exploitation minière et à la prévention des risques miniers après la fin de l'exploitation, enregistré à la présidence de l'Assemblée nationale le 3 mars 1999.

PICOT A., NARBONNE J.-F., GARCIA S., 2013, Chape de plomb sur le site minier de Saint-Félix-dePallières (Gard), Dossier d'information $\mathrm{n}^{\circ} 7$, Association Toxicologie-Chimie, $14 \mathrm{p}$.

RENON A. et JOUVE A., 2014, Pollution minière en France, scandale environnemental en France à la porte des Cévennes, Webdoc Radio France Internationale.

TARDIVON P. et DELAUNAY A. et BESSON A., Contamination par les métaux lourds d'habitants proches d'anciens sites miniers du Gard. Études environnementales sur l'état des sols et nécessité d'assurer un suivi médical, décembre 2016, 68 p.

VAULONT I. et VO VAN QUI J.-L., 2011, Rapport sur « l'indemnisation des dégâts miniers ».

\section{NOTES}

1. Concessions de la "Croix de Pallières ", "Pallières-Gravouillère ", "Saint-Joseph », «Valensole » et « Valleraube ».

2. Association d'Habitants de Logements Éphémères ou Mobiles créée en 2005.

3. ANR EFFIJIE 13-SENV-0001-01, projet «L'effort environnement comme inégalité : justice et iniquité au nom de l'environnement", porté par IRSTEA en collaboration avec le CERTOP (Université de Toulouse), le CURAPS (Université de La Réunion), le GREThA (Université de Bordeaux), le LPED et TELEMME (Aix-Marseille Université).

4. Entretien avec l'auteure, 18 août 2016.

5. Site de l'association Le Filon.

6. BO des associations, modification de l'objet, $\mathrm{n}^{\circ}$ de parution 20110032, 6 août 2011.

7. BO op. cit, $\mathrm{n}^{\circ}$ de parution 20120027,7 juillet 2012

8. Idem, $\mathrm{n}^{\circ}$ de parution 20160010, 27 février 2016.

9. À l'initiative des collectifs Stop au gaz de schiste d'Anduze, BastaGaz Alès, le collectif du Vigan, le collectif Cigalois, le collectif vigilance Hautes Cévennes.

10. En ligne sur le site de Ingénieurs sans frontières http://www.isf-systext.fr/node/934.

11. Entretien avec l'auteure, 18 août 2016.

12. Titre de propriété du GFA, in Maillot avocats associés, "Conclusion devant le tribunal d'Alès ", p. 3.

13. Source : La Gazette, 15-21 décembre 2016.

14. Entretien avec l'auteure, 19 août 2016.

15. Les habitants n'avaient pas été inclus dans le périmètre de l'étude, car informés par la DRIRE et la DDASS de teneurs anormales des polluants dans les sols, ils avaient répondu que «le dépôt n'était occupé que temporairement ", fiche Basol.

16. Entretien avec l'auteure, 18 août 2016.

17. Entretien avec l'auteure, ibid.

18. Entretien avec l'auteure, idem.

19. Entretien avec l'auteure, 19 août 2016.

20. Le propriétaire du GFA est l'un des fondateurs de l'association des objecteurs de croissance.

21. Source : La Gazette, op.cit., p. 9.

22. Générations Futures est une association de défense de l'environnement créée en 1996, agréée par le ministère de l'écologie depuis 2008.

23. Source : Association Générations Futures, 20 janvier 2016.

24. Source : Le Parisien, 25 mars 2016. 
25. Source : site ADAMVM, 11 décembre 2017.

26. Source : Midi Libre, 11 décembre 2014.

27. Entretien de Guy Ethier, vice-président Umicore, 11 février 2016, Site internet d'Umicore.

28. Source : site ADAMVM, op. cit.

29. Source : Compte rendu du Comité de suivi et d'information, op.cit. p. 11.

30. Source : Webdoc RFI 2014.

31. Source : Compte rendu du Comité de suivi et d'information de la Croix de Pallières, 22 juin 2017.

32. Est meuble tout bien qui peut « se transporter d'un lieu à l'autre » (art 528 du Code civil), que ce soit par ses propres moyens (voiture) ou par une intervention extérieure.

33. Entretien avec l'auteure, 19 août 2016.

34. Ordonnance $\mathrm{n}^{\circ} 2011-91$, article L155-6.

35. Circulaire du 25/7/00 sur la mise en œuvre des articles 75-1 à 75-3 du Code minier.

36. Ces quatre principes dégagés par la jurisprudence ont été inscrits dans l'article L110-1 (II, $7^{\circ}$, $2^{\circ}, 3^{\circ}$ et $6^{\circ}$ ) de la loi pour la Reconquête de la biodiversité, de la nature et des paysages, $\mathrm{n}^{\circ}$ 2016-1087 du 8 août 2016.

\section{RÉSUMÉS}

L'étude se focalise sur deux villages ruraux localisés à l'Ouest d'Anduze, dont les populations partagent les nuisances et désordres miniers, de la société belge des mines et fonderies de zinc de la Vieille-Montagne. Est-ce qu'à travers leurs conduites et pratiques de vie sur des sols pollués, les habitants supportent de manière disproportionnée, ou font-ils supporter aux autres les risques toxiques des déchets hérités? Les témoignages recueillis attestent de l'ampleur de la controverse, en révélant les sentiments ambivalents d'incrédulité, de contestation, de révolte et de combat qui les animent face au dévoilement des risques probables et avérés. Dans un premier temps, nous retracerons la socio-histoire de l'implantation des mines sur ce piémont cévenol, moteur de développement depuis la fin du XIX ${ }^{\mathrm{e}}$ siècle jusqu'au lendemain de la seconde Guerre Mondiale suivi d'un déclin de l'activité, puis nous montrerons comment l'apport démographique et le renouveau associatif ont su enrayer le déclin des communes, en raison d'une accessibilité financières aux biens immobiliers pollués. Pour finir, nous examinerons l'imprévisibilité de la gouvernance, dans un cadre politique et juridique complexe de missions cloisonnées et de partage indécis des responsabilités entre l'État, l'industriel, les élus, les institutions.

This study focuses on the two rural villages located west of Anduze, whose populations share nuisances and mining disorders of the Belgian zinc company of Vieille-Montagne. Through their behaviors and living practices on polluted soils, do the inhabitants bear disproportionately, or do they support to others, the toxic risks of legacy waste? The testimonies gathered attest to the extent of the controversy, revealing the ambivalent feelings of incredulity, protest, revolt and struggle that animate them facing the unveiling of probable and proven risks. First of all, we will retrace the socio-history of the implantation of the mines on this piedmont Cevennes, engine of development since the end of the 19th century until the aftermath of the Second World War, then we will show how the demographic contribution and the associative renewal have been able to stop the decline of the communes, because of a financial accessibility to polluted land. Finally, 
we will examine the unpredictability of governance, in a complex political and legal framework of partitioned missions and undecided sharing of responsibilities, between the State, industrialist and mayors.

\section{INDEX}

Keywords : removable and mobile habitats, polluted soils, environmental persistence, hazards and risks, bioaccumulation of toxins, health impacts, economic and ecological losses, causation and attribution of responsibility, environmental remediation

Mots-clés : habitats démontables et mobiles, sols pollués, rémanence dans l'environnement, aléas et risques miniers, bioaccumulation des toxiques, atteintes sanitaires, pertes économique et écologique, causalité et imputation des responsabilité, remédiation environnementale

\section{AUTEUR}

\section{BÉATRICE MÉSIN}

Chargée de recherche en Géographie, CNRS-Telemme-MMSH, Aix-Marseille Université, Mesini@mmsh.univ-aix.fr 